\title{
Essais
}

ESSAIS

Revue interdisciplinaire d'Humanités

Hors-série 3 | 2016

Usages critiques de Montaigne

\section{Ce que les Essais de Montaigne nous apprennent sur la valeur cognitive et morale de la littérature}

\section{Emiliano Ferrari}

\section{(2) OpenEdition}

1 Journals

Édition électronique

URL : http://journals.openedition.org/essais/6874

DOI : $10.4000 /$ essais. 6874

ISSN : 2276-0970

Éditeur

École doctorale Montaigne Humanités

\section{Édition imprimée}

Date de publication : 1 novembre 2016

Pagination : 84-98

ISBN : 979-10-97024-00-0

ISSN : 2417-4211

\section{Référence électronique}

Emiliano Ferrari, «Ce que les Essais de Montaigne nous apprennent sur la valeur cognitive et morale de la littérature », Essais [En ligne], Hors-série 3 | 2016, mis en ligne le 26 février 2021, consulté le 18 mars 2021. URL : http://journals.openedition.org/essais/6874 ; DOl : https://doi.org/10.4000/essais. 6874 


\section{Ce que les Essais de Montaigne nous apprennent sur la valeur cognitive et morale de la littérature}

\section{Emiliano Ferrari}

Un grand pan de la critique littéraire et de la théorie philosophique contemporaines se caractérise par une réflexion stimulante sur la valeur cognitive et morale de la littérature. Des contributions d'Iris Murdoch, Hilary Putnam, Martha Nussbaum et Terence Cave, à celles de Vincent Descombes, Jacques Bouveresse et Marielle Macé, la littérature est considérée comme une archive de récits sur la vie humaine qui nous offre des possibilités d'expérience d'existence, transforme notre environnement cognitif, intensifie notre perception morale et façonne, finalement, notre vie ordinaire ${ }^{1}$.

Dans cette étude, je souhaite me focaliser sur les Essais de Montaigne pour analyser non seulement leur réflexion sur les pouvoirs cognitifs et moraux de la littérature, mais également l'usage cognitif et moral que l'essayiste lui-même fait de littérature, en les faisant converser avec les principales vues qui caractérisent les débats contemporains. À partir de cette confrontation, je propose d'élargir les perspectives historiques et théoriques de ces débats, favorisant en outre une nouvelle appréhension de la valeur littéraire et philosophique des Essais de Montaigne.

1 Je me limite à donner quelques références significatives dans une ample bibliographie : Iris Murdoch, "Philosophy and literature ", in Men of Ideas. Some Creators of Contemporary Philosophy, BBC, Londres, 1978 ; Hilary Putnam, "Literature, science and reflexion ", in Meaning and the Moral Sciences, Boston-Londres, Routledge \& Kegan, 1978, p. $83-94$ (première parution in New Literary, vol. VII, 1975-1976) ; Martha Nussbaum, Love's Knowledge. Essays on Philosophy and Literature, New York, Oxford University Press, 1990 ; Terence Cave, Thinking with Literature. Towards a Cognitive Criticism, Oxford, Oxford University Press, 2016 ; Vincent Descombes, Proust. Philosophie du roman, Paris, Éditions de Minuit, 1987 ; Jacques Bouveresse, La Connaissance de l'écrivain. Sur la littérature, la morale et la vie, Marseille, Agone, 2008 ; Marielle Macé, Façons de lire, manières d'être, Paris, Gallimard, 2011. Voir aussi John Gibson, Fiction and the Weave of Life, Oxford, Oxford University Press, 2007 ; Éthique, litterrature, vie humaine, éd. Sandra Laugier, Paris, Presses Universitaires de France, 2006 ; Carroll Noel, "The Wheel of Virtue: Art, Literature, and Moral Knowledge ", in Art in Three Dimensions, Oxford, Oxford University Press, 2010, p. 201-234. Il est utile de rappeler que ces auteurs se réferent à la littérature « fictionnelle », notamment au roman et à la nouvelle. 


\section{La littérature, la vie et les possibilités humaines}

Quel type de connaissance peut nous offrir la littérature ? Ou mieux : quel type de connaissance Montaigne recherche et trouve dans la littérature ?2 Pour répondre à ces questions, je voudrais d'abord m'appuyer sur deux auteurs contemporains, Hilary Putnam et Martha Nussbaum, pour ensuite revenir à Montaigne.

Dans son article intitulé "Literature, science and reflexion ${ }^{3}$ "(19751976), Hilary Putnam considère que la littérature est une ressource essentielle pour nourrir la réflexion pratique, car faisant travailler nos facultés logiques, imaginatives et affectives, elle nous aide à répondre à la question " comment vivre ? ". La fiction littéraire nous offre une " connaissance de l'homme " (knowledge of man) qui est particulièrement féconde pour la vie morale. Or, de quel type de connaissance s'agirait-il ? Cette connaissance ne semble pas réductible à une connaissance purement empirique, vérifiable et reproductible, mais elle n'est pas non plus une connaissance purement abstraite, ni certes une non-connaissance. Putnam donne deux exemples de textes littéraires très différents : le Voyage au bout de la nuit (1932) de Céline et le Golden Notebook (1962) de Doris Lessing - chaque lecteur peut bien sûr penser à son roman préféré. Pour le philosophe américain, la lecture d'un roman nous montre comment apparait le monde dans une certaine perspective : ce que cela signifie vivre dans ce monde, comment la vie s'y déroule, dans quelles circonstances, quels mobiles animent les êtres humains qui y sont représentés, etc. Lisons ce passage concernant le premier exemple :

If I read Celine's Journey to the End of the Night I do not learn that love does not exist, that all human beings are hateful and hating (even if - and I am sure this is not the case - those propositions should be true). What I learn is to see the world as it looks to someone who is sure that hypothesis is correct. I see what plausibility that hypothesis has; what it would be like if it were true; how someone could possibly think that it is true. But all this is still not empirical knowledge. Yet it is not correct to say that it is not knowledge at all; for being

2 Je ne pense pas que l'on puisse prendre une position dogmatique à propos du type de connaissance que la « littérature " peut nous offrir (scientifique, morale, sociale, politique, etc.). Cela dépend de plusieurs facteurs : le statut littéraire de l'œuvre elle-même, l'intention de l'auteur, du lecteur et le contexte social dans lequel tout cela s'inscrit. Dans le cas de Montaigne, il est clair que son rapport à la littérature - fictionnelle ou non - est façonné par une intentionnalité esthétique, cognitive et (surtout) morale - " Je ne cherche aux livres qu’à m'y donner du plaisir par un honneste amusement ; ou, si j'estudie, je n'y cherche que la science qui traicte de la connoissance de moy mesmes, et qui m’instruise à bien mourir et à bien vivre " (II, 10, 409). Il me paraît évident que le « référentiel » des « livres » est ici la vie. Toutes les références aux Essais renvoient à l'édition Villey-Saulnier publiée par les Presses Universitaires de France.

3 Hilary Putnam, "Literature, science and reflexion ", in Meaning and the Moral Sciences, op. cit., p. 83-94.

$4 \quad$ Ibid., p. 93-94. 
aware of a new interpretation of the facts, however repellent, of a construction that can - I now see - be put upon the facts, however perversely - is a kind of knowledge. It is knowledge of a possibility. It is conceptual knowledge. ${ }^{5}$

D'après Putnam, la fiction littéraire nous offre une connaissance " conceptuelle ", c'est-à-dire une connaissance des possibilités concernant les modes de vie, ou encore, " a vision of how humans behave and of what 'makes them tick $^{6}{ }^{\prime}$ - rappelons que lorsque Montaigne fait l'éloge des poètes, par exemple Térence, c'est justement pour leur capacité à " representer au vif les mouvemens de l'ame et la condition de nos meurs" (II, 10, 411). Ainsi, continue Putnam, ce qui est important pour notre vie morale dans un roman (par exemple The Golden Notebook de Doris Lessing) l'est d'une façon indépendante de sa valeur de vérité, c'est-à-dire de l'exactitude ou l'inexactitude de ses descriptions par rapport au réel ${ }^{7}$.

Dans son important ouvrage Love's Knowledge (1990), Martha Nussbaum propose des vues assez similaires de celles de Hilary Putnam. Très critique de ce qu' elle appelle "The Absence of the Ethical " dans la théorie littéraire de l'époque ${ }^{8}$, Nussbaum souhaite que la critique littéraire retrouve la dimension et le sens moraux des œuvres. En effet, pour l'auteure, la littérature - essentiellement le roman et la nouvelle - nous aide à vivre, car en nous parlant de la condition humaine aux prises avec ses réflexions, ses choix, ses actions et ses émotions individuellement et socialement caractérisées, elle parle aussi de notre vie. Ou mieux, elle offre des "possibilités " pour notre vie. Comme Nussbaum le dit très bien :

It [la littérature] speaks about us, about our lives and choices and emotions, about our social existence and the totality of our connections. As Aristotle observed, it is deep, and conducive to our inquiry about how to live, because it does not simply (as history does) record that this or that event happened; it

5 Ibid., p. 90.

6 "It may seem strange to describe something as real and 'empirical' as a vision of how humans behave and of what 'makes them tick' as conceptual knowledge; but that is all it is unless it is tested, if not scientifically, at least tested in the actual experience of intelligent and sensitive men and women. Thinking of a hypothesis that one had not considered before is conceptual discovery; it is not empirical discovery, although it may result in empirical discovery if the hypothesis turns out to be correct" (Hilary Putnam, op. cit., p. 90, nous soulignons). Lidée que la connaissance offerte par la littérature puisse faire l'objet d'un test ou d'un essai dans la vie concrète du lecteur est essentielle à la conception que Montaigne se fait de la lecture comme " exercice » d'apprentissage à la vie - nous allons y revenir à la fin de cette étude.

7 "There is, however, something Doris Lessing does in The Golden Notebook, which is very important and whose value does not depend on the correctness or incorrectness of her description [...]" (Hilary Putnam, op. cit., p. 91).

8 Martha Nussbaum, Love's Knowledge, op. cit., p. 168-172. Dans ces pages, Nussbaum critique la situation de la théorie littéraire et philosophique des années 1970-1980, qu'elle considère dominée par la tendance " textualiste " - l'on pourrait rajouter également " formaliste " et "structuraliste »-, avec son déni de la portée éthique et sociale de la littérature. 
searches for patterns of possibility - of choice, and circumstance, and the interaction between choice and circumstance - that turn up in human lives with such a persistence that they must be regarded as our possibilities. And so our interest in literature becomes [...] cognitive: an interest in finding out (by seeing and feeling the otherwise perceiving) what possibilities (and tragic impossibilities) life offers to us, what hopes and fears for ourselves it underwrites or subverts. ${ }^{9}$

Comme c'était le cas pour Putnam, Nussbaum met elle aussi en valeur le pouvoir de la littérature comme moyen d'ouverture et d'expérience des possibilités. Plus précisément, la littérature nous offre des modèles et des exemples de possibilités (patterns of possibility) relatifs aux modes de vie, acquérant ainsi une fonction cognitive essentielle dans le développement du jugement éthique du lecteur. Cette connaissance des possibilités humaines est par ailleurs véhiculée dans des formes et des styles perceptifs et imaginatifs qui sont propres à la littérature et qui, contrairement aux abstractions de la philosophie morale, restent proches de la dimension concrète, contradictoire et expérientielle de la vie ordinaire ${ }^{10}$.

Si l'on en vient maintenant aux Essais de Montaigne, nous pouvons nous apercevoir d'une étonnante parenté entre certaines de ses thèses sur la fonction de la "littérature " et celles de Putnam et Nussbaum. Tout particulièrement, nous voyons que dans l'essai I, 21, "De la force de l'imagination ", Montaigne lie de façon manifeste et directe son enquête " anthropologique » et " morale " avec les ressources cognitives que lui offre la littérature, fictionnelle et non fictionnelle.

En l'estude que je traitte de noz moeurs et mouvemens, les tesmoignages fabuleux, pourveu qu'ils soient possibles, y servent comme les vrais. Advenu ou non advenu, à Paris ou à Rome, à Jean ou à Pierre, c'est toujours un tour de l'humaine capacite, duquel je suis utilement advisé par ce recit. Je le voy et en fay mon profit egalement en umbre qu'en corps. Et aux diverses leçons qu'ont souvent les histoires, je prens à me servir de celle qui est la plus rare et memorable. Il y a des autheurs, desquels la fin c'est dire les evenemens. La mienne, si j'y sçavoye advenir, seroit dire sur ce qui peut advenir. (I, 21, 105)

En conclusion du chapitre I, 21, Montaigne s'explique sur l'usage qu'il vient de faire d'exemples et d'anecdotes variés sur la " force de l'imagination ", et caractérise le cadre théorique de ses réflexions comme étant "l'estude [...] de noz moeurs et mouvemens ", c'est-à-dire l'observation et l'analyse

9 Martha Nussbaum, Love's Knowledge, op. cit., p. 171.

10 La littérature contribuerait ainsi, selon Nussbaum, à développer un " jugement éthique " (ethical judgment) conscient de la complexité des situations et des particularités de la vie humaine (Martha Nussbaum, ibid., en particulier l'introduction "Form and Content, Philosophy and Literature ", p. 3-53). 
des actions et des passions humaines ${ }^{11}$. C'est en rapport à cette étude que l'essayiste nous délivre son "mode d'emploi » de la littérature fictionnelle et non fictionnelle. Il s'agit d'un passage crucial qui nécessite, pour être bien compris, d'une exploration de son intertexte. En fait, dans sa réflexion sur la valeur et l'usage du récit et du témoignage écrits ${ }^{12}$, Montaigne semble s'appuyer sur la doctrine de l'invention rhétorique tout en dépassant les limites du discours persuasif pour accentuer la signification cognitive de la diégèse.

Au début de ce passage, Montaigne pose une différence essentielle entre deux formes des « tesmoignages » ou " recit $[s]$ » dont il fait usage : d'une part, « les tesmoignages fabuleux » mais « possibles », d'autre part « les vrais ». Cette différence entre les récits " fabuleux-possibles » et les " vrais » est solidaire de celle entre le "non advenu " et l'" advenu ", ce qui est " en umbre " et " en corps ". Or, ce type de distinction entre le récit des choses fictives, non advenues mais possibles, et des choses vraies et advenues, s'éclaircit à la lumière des notions de la Rhétorique classique et notamment l'argumentum et l'historia. À ce propos, on peut s'appuyer sur un passage du premier livre du De inventione de Cicéron, qui traite des parties du discours persuasif, et

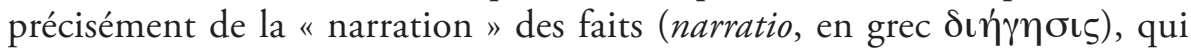
suit l'" exorde » (exordio) et précède la «division" (partitio $)^{13}$.

Selon Cicéron, la «narration » s'organise en deux typologies, l’une concerne les " actions" (negotia), l'autre les "personnes" (personae). La narration qui porte sur les actions se subdivise en " récit légendaire " (fabula), " histoire " (historia) et "fiction" (argumentum). Nous pouvons ainsi schématiser le statut de chaque type de narration selon Cicéron :

- $\quad$ la fabula est une narration qui contient des éléments qui ne sont ni vrais, ni vraisemblables (fabula est in qua nec verae nec veri similes res contenitur) ;

11 Le terme " mouvemens " renvoie bien sûr au movere affectus (émouvoir, exciter les passions) de la rhétorique latine et indique les émotions humaines. Le terme " mœurs ", quant à lui, est clairement défini dans les milieux savants de la deuxième moitié du XVI ${ }^{e}$ siècle, par exemple par le poète Philippe Desportes dans son discours Quelles sont les plus excellentes, les vertus intellectuelles ou les morales: "Quant aux mœurs, ce n'est autre chose à dire que les coustumes et manière de se comporter aux actions et passions humaines [...]" (cité in Édouard Frémy, L'Académie des derniers Valois, Genève, Slatkine Reprints, 1969, réimpression de l'édition de Paris, 1887, p. 232). Selon Marie de Gournay, l'originalité et la singularité de Montaigne s'expriment tout particulièrement dans cette " étude de mœurs " : " Il est bien certain que jamais homme ne dit ni considéra, ce que cettui-ci a dit et considéré, sur les actions et passions humaines : mais il n'est pas certain si jamais homme, lui hors, l'eût pu dire et considérer ", Marie de Gournay, Préface sur les Essais de Michel, Seigneur de Montaigne (1595), in Les Essais, éd. Jean Céard et al., Paris, La Pochothèque, 2001, p. 40.

12 Nous nous intéressons ici au statut du la narration et du témoignage écrits, bien que Montaigne applique ces réflexions à tout récit, qu'il soit écrit ou proféré, lu ou entendu (voir par exemple III, 13, 1081).

13 Cicéron, De l'invention, éd. Guy Achard, Paris, Les Belles Lettres, 1994, I, 19, $27-30$. 
- l'historia raconte un événement ou action qui a lieu dans une époque lointaine (historia est gesta res, ab aetatis nostrae memoria remota) ;

- l'argumentum est la narration d'une histoire inventée mais qui aurait pu arriver : "argumentum est ficta res quae tamen fieri potuit».

Cette division tripartie nous aide à mieux comprendre la position de Montaigne. Dans son étude, il considère comme également utiles et fécondes à ses fins tant les historiae que les argumenta. Les premières, parce qu'elles racontent des actions et des évènements réellement advenus ; les deuxièmes, parce qu'elles récitent des histoires fictives mais qui pourraient advenir. En exposant la nature diégétique et épistémique de ces narrations particulièrement fécondes pour son étude "de noz mœurs et mouvemens", Montaigne en précise aussi leur valeur cognitive, le type de connaissance qu'elles lui apportent.

Les récits fictionnels et non fictionnels, avec la riche variété d'anecdotes, cas et exemples qu'ils contiennent, offrent à l'essayiste une connaissance des possibilités et capacités humaines : " c'est toujours un tour de l'humaine capacite, duquel je suis utilement advisé par ce recit ». Si Montaigne accorde une fonction " référentielle » au texte littéraire, cette fonction n'est pas tant ontologique que logique et conceptuelle ${ }^{14}$, car les textes fictionnels et historiques sont considérés, à cet égard, sur le même plan. Si la valeur de vérité du texte littéraire est donc indépendante de son adéquation au réel, de sa mimesis, c'est aussi parce qu'elle se mesure par rapport à son " utilité " pratique. Pour Montaigne, l'usage de la littérature fictionnelle et/ou non fictionnelle est de type pragmatique, de l'ordre de l'"utile » et du "profit ", car elle contribue à une réflexion de type anthropologique et moral sur les conduites et les passions humaines ${ }^{15}$. Une seule clause semble limiter l'usage de la fiction : comme dans l'argumentum cicéronien, mais pas pour les

14 Montaigne nous paraît néanmoins éloigné des postures structuralistes auxquelles parfois on aimerait le ramener. Si l'essayiste n'a certes pas une conception métaphysique et « fondationaliste » du référentiel textuel - « nous n'avons aucune communication à l'estre » (II, 12, 601) -, il ne croit pas pour autant qu'il n'y a que de "l'écriture ", des " traces ", et qu' « il n'y a pas de hors-texte" (Jacques Derrida, De la grammatologie, Paris, Éditions de Minuit, 1967, p. 227). L'horizon d'attente et de référence par rapport auquel Montaigne apprécie et utilise la littérature reste la vie quotidienne, les actions et les passions humaines, les modes de vie humains réels et possibles, etc. C'est par rapport à la pratique que le langage, écrit ou parlé, prend sens et relief.

15 Cette position a été récemment défendue par Tzvetan Todorov : "L'objet de la littérature est la condition humaine même, celui qui la lit et la comprend deviendra, non un spécialiste en analyse littéraire, mais un connaisseur de l'être humain. Quelle meilleure introduction à la compréhension des conduites et des passions humaines qu'une immersion dans l'œuvre des grands écrivains qui s'emploient à cette tache depuis des millénaires ? ", in La Littérature en péril, Paris, Flammarion, 2007, p. 89. 
mêmes raisons, le récit des choses inventées ne doit pas perdre tout contact avec le réel, il doit garder une apparence de possibilité pour l'être humain, faute de perdre sa valeur conceptuelle et pragmatique ${ }^{16}$.

Mettant sur le même plan les récits fictionnels et historiques, Montaigne paraît assumer à leurs égards une attitude analogue à celle de l'écrivain de romans. Ce dernier, comme le remarque John Searle dans un article pionnier, ne s'engage pas sur la vérité des énoncés qu'il écrits et il est donc exempté de donner des preuves et des évidences de leur vérité factuelle ${ }^{17}$. Si l'on considère le passage du chapitre I, 21 que nous avons analysé, on peut voir qu'il se trouve précédé et suivi par des considérations analogues à celles de Searle :

Les Histoires que j'emprunte, je les renvoye sur la conscience de ceux de qui je les prens. Les discours sont à moy, et se tienent par la preuve de la raison, non de l'expérience : chacun y peut joindre ses exemples : et qui n'en a point, qu'il ne laisse pas de croire qu'il en est, veu le nombre et varieté des accidens. Si je ne comme bien, qu'un autre comme pour moy. [...] Aux exemples que je tire ceans, de ce que j'ay ouï, faict ou dict, je me suis defendu d'oser alterer jusques aux plus legeres et inutiles circonstances. Ma conscience ne falsifie pas un iota, ma science je ne sçay. (I, 21, 105-106).

Intégrant dans son texte les " histoires » et les " exemples » les plus variés, Montaigne n'est pas engagé dans leur vérité factuelle et évènementielle. Il se les approprie comme des " temoignages » qui servent sa réflexion, et qu'il faut juger à l'aune de la "raison ", c'est-à-dire des argumentations et " discours " que Montaigne développe sur un tel ou tel sujet, et non de la " preuve " de l' « experience ». L'essayiste n'est donc aucunement engagé dans la vérité ou fausseté de ces récits, ni dans leur vérification. Comme le fait Plutarque, ainsi l'essayiste n'œuvre pas afin " que ses exemples soient en tout et partout véritables ", mais seulement " qu'ils soient utiles à la postérité, et presentez d'un lustre qui nous esclaire à la vertu»(I, 21, 106, nous soulignons). L'usage

16 Considérant les fictions littéraires comme des "expériences de pensée " qui peuvent nous aider à explorer notre vie morale, Jacques Bouveresse offre un autre point de vue sur le même problème mis en lumière par Montaigne : " Il semble légitime de considérer - écrit Bouveresse que les expériences de pensées ne peuvent pas nous apprendre grand-chose de philosophiquement utile si elles nous entraînent beaucoup trop loin du monde réel. Or les romanciers se posent des problèmes dont la formulation contient des conditions imaginées et qui peuvent être plus ou moins éloignés de la vie réelle ", in La Connaissance de l'écrivain. Sur la littérature, la morale et la vie, op. cit., p. 120).

17 John R. Searle, "The logical status of fictional discourse ", New Literary History, vol. 6, 19741975 (republié dans : Expression and Meaning. Studies in the Theory of Speech Acts Expression and Meaning, Cambridge, Cambridge University Press, 1979, p. 58-89). Donnant comme exemple un passage tiré d'un roman de Iris Murdoch, Searle écrit : "Her utterance is not a commitment to the truth of the proposition [...]. Such a proposition may or may not be true, but Miss Murdoch has no commitment whatever as regards its truth. Furthermore, as she is not committed to its truth, she is not committed to being able to provide evidence for its truth» (p. 62-63). 
cognitif de la littérature a donc une fonction essentiellement pragmatique et incitative : il sert les générations futures en leur offrant des vues qui aident à voir plus clair dans la pratique, à développer un meilleur discernement quant à la "vertu ", c'est-à-dire aux actions et passions.

Qu'ils soient tirés de la poésie, de la philosophie, de l'histoire, des récits de voyage, des compilations des lieux communs, etc., les " exemples", les " histoires ", les " récits " et toutes les narrations que lui offre la tradition littéraire, prennent enfin leur propre sens par rapport au dessein diégétique qui est propre à Montaigne : "Il y a des autheurs, desquels la fin c'est dire les evenemens. La mienne, si j'y sçavoye advenir, seroit dire sur ce qui peut advenir" (I, 21, 105, nous soulignons). S'appuyant sur la distinction aristotélicienne (Poétique) entre « histoire » et " poésie », l'essayiste se place donc du coté du "poète ", de celui qui raconte "les évènements tels qu'ils pourraient arriver » et " qui sont possibles selon la vraisemblance ou la nécessité ${ }^{18}$ ». La valeur cognitive et morale de la "narration " sert cette intention propre aux Essais de Montaigne, et en ce sens on peut dire que l'essayiste fait un usage " humaniste » de la littérature. Pour le dire avec les mots d'un critique contemporain, Montaigne partage " the basic idea that literature represents generalized features of life; that it deals with, to put it simply, how we humans are ${ }^{19}$ ".

\section{L'histoire, la poésie et la connaissance morale}

Comme je viens de montrer, les déclarations montaniennes sur la valeur cognitive et sur l'utilité pratique des narrations, fictionnelles et non fictionnelles, se comprennent en rapport à son " estude [...] de noz mœurs et mouvemens ». Dans le contexte de cette enquête anthropologique et morale, l'historia et l'argumentum valent en tant que " narrations " qui " avisent " utilement l'essayiste quant aux possibilités et capacités humaines, dans le but non pas de dire ce qui est, mais " ce qui peut advenir ${ }^{20}$ ». Ainsi, on peut

18 Aristote, Poétique, in Euvres complètes, éd. P. Pellegrin, Paris, Flammarion, 2014, chap. 9, 1451a 38-b 12, p. 2771. Le texte conclut ainsi : "C’est pourquoi la poésie est plus philosophique et à plus de valeur que l'histoire. En effet, la poésie raconte les événements davantage dans leur généralité ; l'histoire, les événements dans leur particularité. Ce qui est général, c'est le type de choses qu'il appartient à tel type de personne de dire ou de faire, vraisemblablement ou nécessairement [...] ». On rappellera que pour Montaigne "la philosophie n’est qu'une poésie sophistiquée " (II, 12, 517).

19 John Gibson, Fiction and the Weave of Life, Oxford, Oxford University Press, 2007, p. 13.

20 Comme André Tournon l'a également vu, l'usage des exemples chez Montaigne s'affranchit de la pratique rhétorique pour acquérir une dimension réflexive et exploratoire : "En d'autres termes : si l'orateur doit trouver ou imaginer des exemples conformes à son argumentation, le juriste, le casuiste, le philosophe des mours doivent examiner les cas - réels ou fictifs - pour élaborer le jugement qui lui sera conforme ", André Tournon, "Advenu ou non advenu... ", in Montaigne et l'histoire, éd. Claude-Gilbert Dubois, Paris, Klincksieck, 1991, p. 31-38, ici p. 36, nous soulignons. 
dire que la « littérature » est pour Montaigne un réservoir des récits qui, nous montrant la variété des expériences et des pratiques humaines, développe notre capacité de comprendre les formes possibles des comportements et des émotions de nos semblables. Mais comment cette connaissance et expérience des possibilités humaines se réalisent-t-elles effectivement dans les Essais ? Je me bornerai ici à considérer un chapitre des Essais où Montaigne fait interagir dans ce sens deux ressources narratives particulières, qu'il dit privilégier entre toutes dans sa quête de "la connaissance " de "l'homme en général " : l'histoire et la poésie ${ }^{21}$. Mais avant d'en venir à cette étude de cas, il est utile de présenter brièvement quelques passages théoriques des Essais consacrés à la valeur et à l'usage cognitifs et moraux de l'histoire et de la poésie :

Les Historiens sont ma droitte bale : ils sont plaisans et aysez; et quant et quant l'homme en general, de qui je cherche la cognoissance, y paroist plus vif et plus entier qu'en nul autre lieu, la diversité et verité de ses conditions internes en gros et en destail, la varieté des moyens de son assemblage et des accidents qui le menacent. (II, 10, 416)

Plutarque, Hérodote, Quinte-Curce, César, Tite-Live, Francesco Guicciardini, Paolo Giovio... Il est impossible d'évoquer ici la richesse des lectures historiques de Montaigne ${ }^{22}$. Mais loin d'être bornée à sa valeur rhétorique, comme réservoir d'exemples pour le discours persuasif23, l'histoire est appréciée par sa contribution cognitive, pour ce qu'elle nous fait connaître de l'homme " en general ${ }^{24}$. Plus précisément, l'histoire - c'est-àdire les exemples, les récits, les témoignages, etc. - nous offre une vision de l'être humain qui évite toute simplification hâtive et uniformité abstraite, respectant les détails concrets, les conditions particulières et variables, internes et externes, qui caractérisent sa conduite, ses choix, ses émotions.

La poésie, des classiques gréco-latins jusqu'à Torquato Tasso, est hautement appréciée par Montaigne pour sa valeur cognitive et pour sa capacité de montrer l'être humain dans la complexité et variété de ses actions et

21 Il s'agit d'une affinité élective que Montaigne confesse dans l'essai I, 26 : "L'Histoire, c'est plus mon gibier, ou la poesie, que j'ayme d'une particuliere inclination " (I, 26, 146). Nous n'oublions pas, bien entendu, que la lecture de l'histoire et de la poésie a aussi une valeur esthétique pour Montaigne, mais cela dépasse le sujet de notre étude.

22 Sur la complexité du rapport montanien à l'histoire, sur la richesse sémantique de ce mot dans les Essais, sur les multiples formes de narration historique - "exemples ", " tesmoignage ", " recit ", " conte ", etc. -, on verra les études réunies dans Montaigne et l'histoire, op. cit.

23 Voir la longue digression sur l'histoire dans l'Orateur de Cicéron (II, 12, 51-II, 15, 64).

24 Sur ce point, voir notre ouvrage Montaigne, une anthropologie des passions, Paris, Classiques Garnier, 2014, p 135-152. 
passions ${ }^{25}$. C’est ainsi que Montaigne juge la poésie de Térence : «Quant au bon Terence, la mignardise et les graces du langage Latin, je le trouve admirable à representer au vif les mouvemens de l'ame et la condition de nos mœurs ; à toute heure nos actions me rejettent à luy " (II, 10, 411). Si dans histoire l'homme " paroist plus vif et plus entier qu'en nul autre lieu ", la bonne poésie, quant à elle, a le pouvoir de "representer au vif » les mours des êtres humains. Montaigne semble ici lier les performances cognitives de la poésie et de l'histoire à leurs qualités stylistiques et esthétiques. Tout particulièrement, l'essayiste évoque la théorie de la "vive représentation " (enargeia), qui occupe une place centrale dans la poétique et la rhétorique renaissantes ${ }^{26}$, pour la mettre moins au service du persuadere que du docere. La description vive et détaillée nous montre, nous apprend et nous découvre "nos actions » et " mouvemens ». Par ailleurs, paradoxalement, bien qu'elle réponde à une logique fictionnelle et créatrice (poïesis), la poésie semble pour Montaigne mieux respecter les conditions réelles de notre vie morale. Contrairement aux prétentions de l'éthique stoïcienne, qui voudrait forcer "nos conditions naturelles » dans les grilles de son système, la poésie adopte un point de vue humain et n'hésite pas à représenter un héros comme Énée aux prises avec ses émotions ordinaires : «Les poetes qui feignent tout à leur poste, n'osent pas descharger seulement des larmes leurs heros: Sic fatur lachrymans, classique immittit habenas " (II, 2, 346) ${ }^{27}$.

25 Voir aussi Olivier Guerrier, "Quand les poètes feignent ": "fantasie " et fiction dans les Essais de Montaigne, Paris, H. Champion, 2002 ; et le numéro spécial des Montaigne Studies sur "Montaigne et la poésie ", éd. F. Goyet, vol. XVIII, 2006. On rappellera que Montaigne était un grand lecteur et amateur de poésie : sur ces goûts personnels voir par exemple II, 10, 410-412. Pour l'essayiste, la poésie est aussi source d'une intense expérience affective qui comme le veut la doctrine classique du sublime - s'apparente au ravissement et à l'extase : voir I, 37, 231-232.

26 Voir Agnès Rees, "Poétiques de la 'vive représentation' de Marco Girolamo Vida (1527) à Jacques Peletier du Mans (1555) », Italique, vol. XII, 2009, p. 93-122 ; Perrine Galand, Les Yeux de l'éloquence. Poétiques humanistes de l'évidence, Orléans, Paradigme, 1995 ; ClaudeGilbert Dubois, "Itinéraire et impasses de la 'Vive Représentation' au XVI' siècle ", in La Littérature de la Renaissance. Mélanges offerts à Henri Weber, Genève, Slatkine, 1984, p. 405-25. Au livre VI de son Institution oratoire (VI, 2, 32), au milieu des réflexions concernant la production des émotions (circa movendo adfectus), Quintilien définit l'enargeia comme étant une description qui nous représente les choses dans tous leurs détails et circonstances, comme si elles étaient devant nos yeux (in oculis). Pour Quintilien, la vive description (enargeia, evidentia, illustratio) relève à la fois de l'ornement (ornatus) et de la narration (narratio), et est associée à l'ekphrasis et à la production des affects dans l'auditoire (voir P. Galand, Les Yeux de l'éloquence. Poétiques humanistes de l'évidence, op. cit., p. 100).

27 Pour les vers latins : Virgile, Enéide, 6, 1. Ces vers font la transition avec la fin du chant 5 : en abordant Cumes, Énée pleure la mort de son timonier Palinure, tombé dans la mer victime du dieu Sommeil. 
Je voudrais à présent évoquer un cas manifeste de l'interaction entre l'historie, la poésie et la connaissance anthropologico-morale, marrêtant sur le chapitre I, 38, "Comme nous pleurons et rions d'une mesme chose ${ }^{28}$ ". La réflexion de l'essai se développe en articulant les uns après les autres exemples historiques, expériences personnelles, vers poétiques et énoncés philosophiques. Parmi les auteurs et les textes sollicités : les Vies de Plutarque, les Histoires d'Hérodote, les Annales de Tacite, les Poèmes de Catulle, le De rerum natura de Lucrèce, la Pharsale de Lucain, les Chroniques de Froissart, les Annales de Gilles, le Chansonnier de Pétrarque, et la Théologie naturelle de Raimond Sebond. Tous ces "teismognages ", historiques ou fictionnels, se présentent comme autant de variantes d'un même comportement humain qui intéresse Montaigne, et qui consiste dans cette réaction émotive apparemment incohérente et contradictoire qui caractérise la vie humaine. Pour ne donner que quelques exemples, comment expliquer que César pleure la tête de son ennemi Pompée, ou que quelqu'un s'attriste au départ d'un voyage qu'il souhaite, ou qu'une jeune fille éprise d'amour rejoint l'être aimé en larmes souffrant de quitter sa famille, ou encore que Montaigne gronde son valet pour ensuite se montrer bienveillant ? Dans ce cas précis, la contribution cognitive de la poésie est intégrée dans la réflexion pour être ensuite dépassée - cela est aussi possible ${ }^{29}$-, car ses conclusions apparaissent à Montaigne vraies mais partielles. Pleurer pour la mort de son ennemi n'est pas forcément, comme le suggèrent Lucain et Pétrarque ${ }^{30}$, un acte de dissimulation qui cacherait, derrière les larmes, un sentiment de joie :

Car, bien que, à la verité, la pluspart de nos actions ne soient que masque et fard, et qu'il puisse quelquefois estre vray, Haeredis fletus sub persona risus est, si est-ce qu'au jugement de ces accidens il faut considerer comme nos ames se trouvent souvent agitées de diverses passions. (I, 38, 234; pour la citation latine: Aulu-Gelle, Nuits Attiques, XVII, 14, 4)

La vérité des poètes opère ici comme une ressource cognitive qui doit être replacée dans un contexte plus élargi, prenant en compte la part de complexité et d'irréfléchi de notre vie psychique. L'intégration d'autres exemples, tirés de

28 Bien entendu, presque tous les chapitres des Essais peuvent être étudiés dans ce sens, et non seulement relativement à l'usage de l'histoire et de la poésie, mais également d'autres genres tels que la comédie, la satire, la tragédie, etc.

29 Si Montaigne conteste ici les conclusions des poètes cela n’implique pas qu'il les dévalorise pour autant : ils lui offrent des éléments de compréhension qu'il considère valides mais seulement dans certaines circonstances. De fait, Montaigne s'attache à réviser la vérité des énoncés poétiques pour les intégrer dans une vision plus complexe et élargie des comportements qui font l'objet de son analyse. Pour une analyse détaillée du chapitre I, 38, et de ses aperçus psychologiques et moraux, je me permets de renvoyer à mon ouvrage Montaigne, une anthropologie des passions, op. cit., p. 143-152.

30 Montaigne cite le Chansonnier de Pétrarque (CII, 9-11) et sa source latine, la Pharsale de Lucain, où les larmes de César sont considérées comme insincères, cachant en vérité un sentiment de joie (IX, 1037-1039). 
Catulle, Hérodote et Lucrèce, prépare à une nouvelle compréhension de ce comportement affectif. L'expérience des émotions conflictuelles et opposées que l'on peut éprouver devant la même personne ou le même événement, peut ainsi être réinterprétée selon des énoncés généraux que Montaigne formule dans le chapitre.

1) "Nulle qualité nous embrasse purement et universellement " (I, $38,234)$;

2) «Ainsin eslance nostre ame ses pointes diversement et imperceptiblement " (ibid., 235) ;

3) «Il n'y a rien de changé, mais nostre ame regarde la chose d'un autre oeil, et se la represente par un autre visage : car chaque chose a plusieurs biais et plusieurs lustres" (ibid.).

La conduite d'un être humain n'est jamais réductible à une seule " qualité ", à une propriété univoque et générale. Les comportements individuels sont complexes, car leurs mobiles psychiques sont changeants et peuvent se manifester de façon prompte et imperceptible. Les émotions, par ailleurs, dépendent en bonne partie de nos manières de regarder les choses, qui se trouvent au milieu d'un réseau de relations et liens leur donnant des sens et des aspects multiples et contingents. Si l'amour pousse résolument la jeune fille vers l'être aimé, le départ de sa famille lui montre tout ce qu'elle abandonne, les liens, les accointances, les habitudes : ces images «saissent [son] imagination, et la passionnent pour l'heure ${ }^{31}$ ".

Les récits historiques et les vers poétiques permettent ainsi à Montaigne d'analyser le comportement humain restant près des détails et des contextes particuliers, produisant un jugement éthique qui relève d'une généralité et universalité concrètes et incarnées. Ainsi, par rapport au procédé schématique de la philosophie morale, qui comprend la multiplicité des conduites humaines en les ordonnant par des critères d'exactitude et des lois générales $^{32}$, l'approche montanienne privilégie l'observation des situations, la description des détails, l'analyse des circonstances accidentelles, visant à une compréhension du particulier (qu'il appelle "jugement») qui n'est pas

31 Voir aussi les détails qui suivent l'exemple de César et Pompée (I, 38, 233) et celui de la "vengence d'une injure " (ibid., 235).

32 C'est exactement ce que Montaigne refuse de faire. Parlant de son étude de la vie humaine, il dit de ne pas vouloir : "renger cette infinie varieté d'actions, si diverses et si descoupées, à certains genres et chapitres, et distribuer distinctement [s] es partages et divisions en classes et regions cogneues [...]. Je laisse aux artistes, et ne sçay s'ils en viennent à bout en chose si meslée, si menue et fortuite, de renger en bandes cette infinie diversité de visages, et arrester nostre inconstance et la mettre par ordre. Non seulement je trouve mal-aisé d'attacher nos actions les unes aux autres, mais chacune à part soy je trouve mal-aysé de la designer proprement par quelque qualité principalle, tant elles sont doubles et bigarrées à divers lustres " (III, 13, 1076-1077). Voir aussi le début du chapitre II, 1. 
la simple application aux contenus de l'expérience des règles ou principes formulés avant l'expérience même. De cette façon, le jugement éthique montanien peut suivre le mouvement "inegal, irregulier et multiforme " de la vie (III, 3, 819), respecter les aspects et les contextes significatifs des situations morales, s'efforçant de préserver le juste équilibre entre la singularité et la généralité.

\section{Conclusion}

Effaçant la différence entre les narrations fictionnelles et non fictionnelles, en suspendant leur valeur de vérité à une opération référentielle de type conceptuel et pragmatique, les Essais de Montaigne offrent une contribution importante au débat contemporain sur la valeur cognitive et morale de la littérature. Dans le sillage du topos aristotélicien, Montaigne exprime de façon marquante une vision de la littérature qui en accentue, comme le font par exemple Putnam et Nussbaum, sa capacité de connaissance et d'expérience des possibilités et des modes de vie humains, à la recherche d'un jugement éthique qui joint le particulier à l'universel, l'exemple à la règle ${ }^{33}$.

L'insertion d'exemples historiques et de récits fictionnels permet à la réflexion morale montanienne d'éviter le piège du dogmatisme ou de "l'idéalisme moral ${ }^{34}$ " : c'est-à-dire, la tentation de simplifier les problèmes moraux, de les résoudre dans des principes, des leçons et des dogmes ${ }^{35}$. Les histoires et les récits fictionnels (poétiques, tragiques, etc.), jouent pour Montaigne la même fonction que la nouvelle ou le roman chez les auteurs contemporains que nous avons évoqués. Par leur forme ouverte et mouvante, par leur attention aux détails, aux circonstances dans lesquelles prennent forme nos actions, nos choix et nos émotions, les exemples refrènent «l'esprit de système $^{36}$ " du discours philosophique, l'obligeant à se confronter avec la contingence du réel et sa résistance aux réductions hâtives, aux « jugemens en gros " qui sont toujours « laches et imparfaicts " (III, 8, 943).

33 Voir plus haut, notes 5 et 9. Une vue analogue est proposée par Frédérique Leichter-Flack: "À la différence des scénarios hypothétiques artificiellement élaborés par les philosophes pour servir de support à leur réflexion éthique, les fictions littéraires sont le reflet le plus fidèle - en contexte - de la réalité possible, celle pour laquelle on doit se fixer des règles de conduite. Mais comment dégager des règles à partir de cas toujours particuliers, toujours spécifiques ? ", in $L e$ Laboratoire des cas de conscience, Paris, Alma, 2012, p. 12-13.

34 J'emprunte cette expression à J. Bouveresse, La Connaissance de l'écrivain. Sur la littérature, la morale et la vie, op. cit., p. 133, 137, 138-139.

35 Voir à ce propos les remarques très pertinentes de Jean-Yves Pouilloux, Montaigne : l'éveil de la pensée, Paris, H. Champion, 1995, p. 17-20.

36 André Tournon, "Advenu ou non advenu... ", art. cit., p. 37. 
Par leur manière de penser, représenter et examiner les actions et les passions humaines, s'appuyant sur les ressources descriptives et narratives de l'histoire et de la poésie, entre autres, les Essais nous offrent - comme le suggère Terence Cave - un exemple paradigmatique du travail de la littérature ${ }^{37}$, de la capacité du texte non seulement de divertir son lecteur, mais d'en exercer également toutes ses facultés cognitives (jugement, imagination, perception, mémoire) ${ }^{38}$. "Les livres - écrit Montaigne - m'ont servi non tant d'instruction que d'exercitation » (III, 12, 1039). Cet exercice est une école ou un laboratoire de réflexion morale, qui développe en nous un jugement plus fin, plus attentif aux situations éthiques toujours nouvelles et imprévues que la vie nous offre.

Je crois enfin, que la contribution des Essais au débat contemporain sur le sens et l'usage cognitifs et moraux de la littérature, est tout à fait fondamentale et mériterait d'être étudiée et exploitée davantage. Les Essais, texte hybride, instable et multiforme, conçu comme " un registre des essais de ma vie " écrit Montaigne (III, 13, 1079), sont sans doute l'un des meilleurs exemples d'une réflexion philosophique qui se créé d'elle-même, en explorant les rapports multiples et étroits entre la littérature, la connaissance morale et la vie ${ }^{39}$.

Emiliano Ferrari

Université Jean Moulin Lyon 3

\section{Résumé}

De Martha Nussbaum à Terence Cave, la critique littéraire et la théorie philosophique contemporaines s'interrogent sur la valeur cognitive et morale de la littérature. Fondant leur enquête anthropologique et morale sur un usage cognitif et pragmatique de la littérature fictionnelle et non fictionnelle, les Essais de Montaigne nous offrent un exemple marquant des rapports étroits et féconds entre la littérature, la philosophie et la vie.

37 Terence Cave, Thinking with Literature. Towards a Cognitive Criticism, op. cit., p. 5 : «Essais are arguably among the most powerful and comprehensive of written instruments for inflecting the cognitive environment of the reader. In that sense, his book is a paradigm of literature itself : literary works make you think differently ". Je tiens à remercier T. Cave pour avoir gentiment mis à ma disposition les épreuves de son livre : la perspective théorique et critique qui y est développée a été une source d'inspiration pour mon étude.

38 Et bien sûr, la littérature exerce les capacités affectives du lecteur. Ce thème, très important, dépasse largement les limites de mon enquête. On pourra néanmoins rappeler que les émotions sont étroitement liées à nos fonctions cognitives et imaginatives, et ont une valeur constitutive de nos jugements éthiques. Sur ce point, on pourra voir les analyses de Martha Nussbaum, Love's Knowledge, op. cit., chapitres 2, 11, 12.

39 Pour une analyse de la valeur cognitive de l'essai montanien en tant que mode de pensée et forme d'écriture, nous renvoyons à notre article "'A knowledge broken': Essay Writing and Human Science in Montaigne and Bacon ", Montaigne Studies, vol. XXVIII, 2016, p. 211-221. Sur le lien essentiel entre la structure formelle et le contenu éthique de l'essai, voir aussi Jean-Yves Pouilloux, Montaigne : l'éveil de la pensée, op. cit., p. 210-213. 


\title{
Mots-clés
}

Montaigne, littérature, philosophie morale, fiction, cognition, émotions.

\begin{abstract}
From Martha Nussbaum to Terence Cave, contemporary literary criticism and philosophy question the moral and cognitive value of literature. Founding their anthropological and moral investigation on a cognitive and pragmatic usage of fictional and non-fictional literature, Montaigne's Essais offers a striking example of the productive and close relations between literature, philosophy and life.
\end{abstract}

\section{Keywords}

Montaigne, literature, moral philosophy, fiction, cognition, emotions. 